\title{
The effects of polygenic risk for psychiatric disorders and smoking behaviour on psychotic experiences in UK Biobank
}

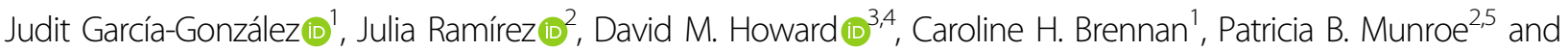 \\ Robert Keers ${ }^{1}$
}

\begin{abstract}
While psychotic experiences are core symptoms of mental health disorders like schizophrenia, they are also reported by $5-10 \%$ of the population. Both smoking behaviour and genetic risk for psychiatric disorders have been associated with psychotic experiences, but the interplay between these factors remains poorly understood. We tested whether smoking status, maternal smoking around birth, and number of packs smoked/year were associated with lifetime occurrence of three psychotic experiences phenotypes: delusions $(n=2067)$, hallucinations $(n=6689)$, and any psychotic experience (delusions or hallucinations; $n=7803$ ) in 157,366 UK Biobank participants. We next calculated polygenic risk scores for schizophrenia $\left(\mathrm{PRS}_{\mathrm{SCZ}}\right)$, bipolar disorder $\left(\mathrm{PRS}_{\mathrm{BP}}\right)$, major depression $\left(\mathrm{PRS}_{\mathrm{DEP}}\right)$ and attention deficit hyperactivity disorder (PRS ${ }_{\mathrm{ADHD}}$ ) in 144,818 UK Biobank participants of European ancestry to assess whether association between smoking and psychotic experiences was attenuated after adjustment of diagnosis of psychiatric disorders and the PRSs. Finally, we investigated whether smoking exacerbates the effects of genetic predisposition on the psychotic phenotypes in gene-environment interaction models. Smoking status, maternal smoking, and number of packs smoked/year were associated with psychotic experiences $\left(p<1.77 \times 10^{-5}\right)$. Except for packs smoked/year, effects were attenuated but remained significant after adjustment for diagnosis of psychiatric disorders and PRSs $(p<$

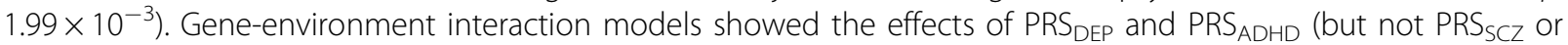
$\mathrm{PRS}_{\mathrm{BP}}$ ) on delusions (but not hallucinations) were significantly greater in current smokers compared to never smokers $(p<0.002)$. There were no significant gene-environment interactions for maternal smoking nor for number of packs smoked/year. Our results suggest that both genetic risk of psychiatric disorders and smoking status may have independent and synergistic effects on specific types of psychotic experiences.
\end{abstract}

\section{Introduction}

Psychotic experiences, such as hallucinations (unreal visual or auditory perceptions) and delusions (unreal beliefs or impressions, i.e. conspiracy against self, unreal communications or signs), are reported by $5-10 \%$ of the general

Correspondence: Judit García-González (j.garciagonzalez@qmul.ac.uk) ${ }^{1}$ School of Biological and Chemical Sciences, Queen Mary University of London, London E1 4NS, UK

${ }^{2}$ Clinical Pharmacology, William Harvey Research Institute, Barts and The London School of Medicine and Dentistry, Queen Mary University of London, London EC1M 6BQ, UK

Full list of author information is available at the end of the article

These authors contributed equally: Patricia B. Munroe, Robert Keers population $^{1}$. Psychotic experiences are core symptoms of severe mental disorders, such as schizophrenia, and therefore may be an indicator of risk for mental health problems even in apparently healthy individuals. Understanding their aetiology could help identify individual susceptibility for different mental health outcomes and develop tailored prevention and intervention strategies.

Genetic factors influence psychotic experiences, explaining between $30 \%$ and $50 \%$ of the variance in twin studies ${ }^{2,3}$ and common genetic variation explaining between $3 \%$ and $17 \%$ of the variance in molecular genetic studies ${ }^{4,5}$. Twin studies also suggest that the genetic component of psychotic

\section{(c) The Author(s) 2020}

(c) (i) Open Access This article is licensed under a Creative Commons Attribution 4.0 International License, which permits use, sharing, adaptation, distribution and reproduction cc) in any medium or format, as long as you give appropriate credit to the original author(s) and the source, provide a link to the Creative Commons license, and indicate if changes were made. The images or other third party material in this article are included in the article's Creative Commons license, unless indicated otherwise in a credit line to the material. If material is not included in the article's Creative Commons license and your intended use is not permitted by statutory regulation or exceeds the permitted use, you will need to obtain permission directly from the copyright holder. To view a copy of this license, visit http://creativecommons.org/licenses/by/4.0/. 
experiences is shared with psychiatric disorders such as schizophrenia, bipolar disorder, depression and attention deficit hyperactivity disorder (ADHD) ${ }^{6}$. Large genome-wide studies of psychotic experiences in UK Biobank (UKB) confirm these findings: polygenic risk scores (PRS) of schizophrenia, bipolar disorder, depression, and ADHD explain $0.11 \%, 0.06 \%, 0.32 \%$ and $0.05 \%$ of the variance in psychotic experiences, respectively ${ }^{7}$. Linkage disequilibrium score regression (LDSC) indicates the genetic correlation estimates between psychotic experiences and schizophrenia, bipolar disorder, depression and ADHD are 0.21, 0.15, 0.46 and $0.24^{7}$.

In addition to genetic influences, environmental factors explain between $50 \%$ and $70 \%$ of the variance in psychotic experiences $^{3}$. Smoking is one of the best established environmental risk factors associated with the occurrence of psychotic experiences in case-control studies of schizophrenia and bipolar disorder, as well as in population-based samples. Longitudinal studies suggest that continued smoking is a causal factor for psychotic experiences among people with the first episode of psychosis ${ }^{8}$, schizophrenia, and bipolar disorder patients ${ }^{9}$. The observation of a doseresponse effect in prospective studies ${ }^{10-13}$, together with recent Mendelian randomization studies ${ }^{14}$, further support a causal effect of smoking on the occurrence of psychotic experiences. In addition, the associations between lifetime tobacco use and subsequent psychotic experiences ${ }^{15,16}$ in the general population remained significant after adjusting for other psychiatric disorders ${ }^{16}$, suggesting that smoking may lead to psychotic experiences independently from mental health status.

Associations between other smoking phenotypes and psychotic experiences are less well-established. There is much less evidence for an increased risk of psychotic experiences in former smokers, leading some to suggest that the effects of smoking are reversible ${ }^{17,18}$. However, it is still unclear whether these findings are false positives resulting from a lack of power in relatively small samples. Maternal smoking during pregnancy is associated with psychotic experiences in offspring ${ }^{19}$. However withinfamily studies suggest that once familial factors are taken into account, maternal smoking during pregnancy has no effect on the risk of psychotic experiences ${ }^{20}$.

Despite evidence showing associations between genetic risk for psychiatric disorders and psychotic experiences ${ }^{6,7}$, and a number of studies showing associations between smoking and psychotic experiences ${ }^{17,21-23}$, the interplay between smoking and genetic risk on psychotic experiences remains unknown. There are studies showing that PRSs for psychiatric disorders are associated with smoking $^{24-27}$. This suggests that smoking may not be only a casual factor in the development of psychotic experiences, but that common genetic factors may affect both smoking and psychotic experiences. However, no studies have tested whether PRSs for psychiatric disorders and smoking make independent contributions to psychotic experiences. Significant gene-environment interactions (GxE) have been reported for several psychiatric traits including schizophrenia and depression ${ }^{28-30}$, but studies are yet to explore whether there is an interaction between smoking and PRSs for psychiatric disorders on psychotic experiences. That is, whether the effects of genetic risk are greater for individuals exposed to smoking.

Our first objective was to investigate whether smoking status (current, former or never smoker), number of packs smoked per year and maternal smoking around birth were associated with lifetime delusions or hallucinations or any psychotic experience (delusions or hallucinations) in the UKB cohort. We also tested whether the effects of smoking remained significant after adjustment for diagnosis of depression, ADHD and psychotic disorders (schizophrenia, mania, hypomania, bipolar or manic depression, and schizotypal or delusional disorders), as well as genetic risk for these disorders, captured by PRSs for depression $\left(\mathrm{PRS}_{\mathrm{DEP}}\right)$, ADHD ( $\left.\mathrm{PRS}_{\mathrm{ADHD}}\right)$, schizophrenia $\left(\mathrm{PRS}_{\mathrm{SCZ}}\right)$ and bipolar disorder $\left(\mathrm{PRS}_{\mathrm{BP}}\right)$. Our second objective was to investigate whether the effects of smoking on psychotic experiences were exacerbated by genetic risk by testing additive and multiplicative GxE between these variables. We interrogated whether being a current, former or never a smoker; smoking more packs of cigarettes per year; or being exposed to maternal smoking around birth influenced the effects of PRSs on having hallucinations, delusions, or any of the two. When significant interactions between smoking and PRS were identified, we carried out sensitivity analyses excluding people with psychotic disorders. An overview of the study design is provided in Fig. 1.

\section{Methods \\ UK Biobank sample}

The sample was drawn from UKB, a population-based cohort from the United Kingdom with $\sim 500,000$ participants. From those, 157,366 participants completed an online mental health questionnaire. A detailed description of the mental health questionnaire has been provided elsewhere ${ }^{31}$. Ethical approval was provided by UKB (Application ID\#42423) and all participants gave written consent.

\section{Measures \\ Psychotic experiences}

Psychotic experiences were categorised based on the Composite International Diagnostic Interview included in the mental health questionnaire. Three outcomes were defined: delusions, hallucinations, and any psychotic experience. Delusions were ascertained as individuals reporting lifetime delusions of reference and/or persecutory delusions (Field IDs: 20474, 20468). Hallucinations 

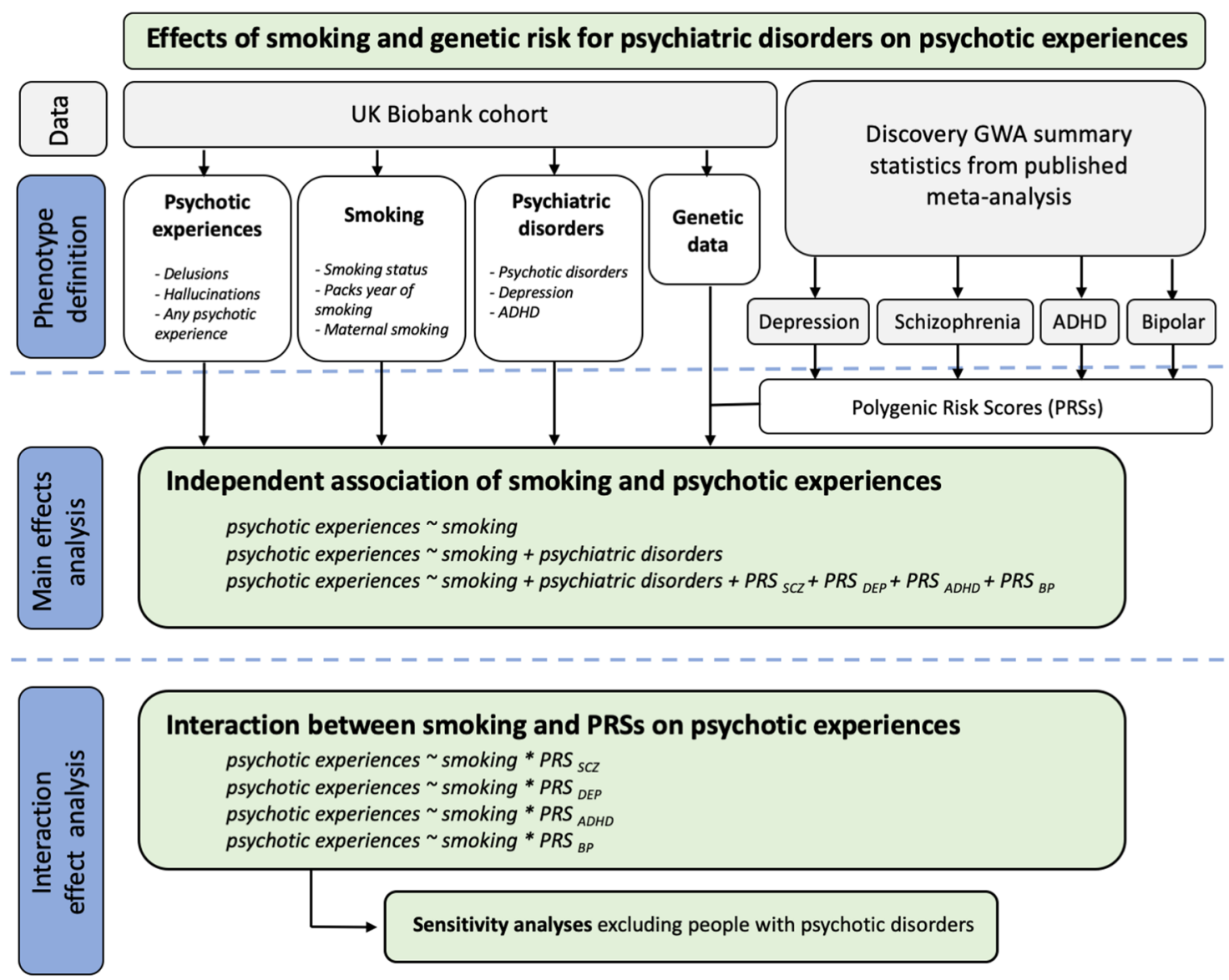

Fig. 1 Detailed flowchart of the analytical approach. UKB UK Biobank cohort. PRSs polygenic risk scores for schizophrenia (PRS $S_{S C Z}$ ), depression $\left(P R S_{D E P}\right), A D H D\left(P R S_{A D H D}\right)$ and Bipolar disorder $\left(P_{B P S}\right)$. GWA genome-wide association. ADHD Attention deficit hyperactivity disorder.

were ascertained as individuals reporting lifetime visual and/or auditory hallucinations (Field IDs: 20471, 20463). Any lifetime psychotic experiences were ascertained as a positive response to any of the four symptom questions (Field IDs: 20474, 20468, 20471, 20463). Controls were defined as individuals that did not endorse lifetime psychotic experiences. A more detailed description of the outcome variables is provided in the Supplementary Tables 1-3.

\section{Smoking}

Three smoking outcomes were defined using the UKB baseline questionnaire: smoking status (Field ID 20116), pack years of smoking (Field ID 20161) and maternal smoking around birth (Field ID 1787). The smoking status phenotype consisted of never smoker, former smokers and current smokers. Pack years of smoking were calculated for former and current smokers and it was defined as the number of cigarettes smoked per day, divided by twenty, multiplied by the number of years of smoking. Cases for maternal smoking were defined as individuals endorsing
'Yes' for the question 'Did your mother smoke regularly around the time when you were born?'.

\section{Other psychiatric disorders}

Lifetime depression, ADHD, schizophrenia, mania, hypomania, bipolar or manic depression, and any other type of psychotic disorder were defined using both the UKB baseline and mental health questionnaires (Supplementary Tables 4 and 5).

\section{UKB genetic dataset}

The data release contained 488,377 individuals genotyped on either the UKB Axiom or the UK BiLEVE Axiom arrays. Genotype data were imputed centrally by UKB with IMPUTE2 using the Haplotype Reference Consortium panel ${ }^{32}$. UKB also provided metrics for quality control that were used to exclude individuals with poor genotype call rate $(>5 \%)$ and discordance between the self-reported sex and the sex inferred from the genotypes. The first two principal components provided by UKB were used in a $\mathrm{k}$-means clustering algorithm ${ }^{33}$ to identify 
a genetically homogeneous subsample. Related individuals were identified for each phenotype separately using the $R$ package 'ukbtools ${ }^{34}$ and one individual from related pairs/trios were excluded (kinship coefficient $>0.0884$ ). Details on the quality control (QC) of genetic data and sample sizes after QC exclusion are provided in Supplementary Table 6.

\section{PRSs}

We calculated PRS $\mathrm{SCZ}_{\mathrm{SC}}, \mathrm{PRS}_{\mathrm{DEP}}, \mathrm{PRS}_{\mathrm{ADHD}}$ and $\mathrm{PRS}_{\mathrm{BP}}$ for each UKB participant using the software PRSice- $2^{35}$ and summary statistics from four recent genome-wide association studies GWAS of these disorders (Supplementary material) $)^{36-39}$. Logistic regression models were used to explore the association between $\mathrm{PRS}_{\mathrm{SCZ}}, \mathrm{PRS}_{\mathrm{DEP}}$, $\mathrm{PRS}_{\mathrm{ADHD}}$ and $\mathrm{PRS}_{\mathrm{BP}}$ and psychotic experiences using PRSice- ${ }^{35}$. Analyses included the first ten principal components and genotyping batch as covariates.

\section{Main effect analyses}

Regression models were constructed to assess the association between maternal smoking, smoking status and number of packs smoked per year and psychotic experiences. To test whether the association between smoking and psychotic experiences was independent of diagnosis for psychiatric disorders and genetic predisposition, models were rerun adjusting for: diagnosis for psychotic disorders, depression, and ADHD, as well as the PRS with the highest predictive ability ( $p$-value threshold $=0.2$ ) on psychotic experiences.

\section{Interaction effect analyses}

We explored whether there were GxE between each of the PRSs and smoking on psychotic experiences (i.e. whether the effects of genetic risk differed among current, former or never smokers, among smokers that consumed more packs of cigarettes per year, and among individuals whose mothers smoked around time of participants' birth). Interactions were tested on the multiplicative and additive scales using logistic and linear regressions, respectively. Interactions on the multiplicative scale assess whether the joint effect of the PRS and smoking is greater than the product of their individual effects. Interactions on the additive scale assess whether the joint effect of smoking and the PRS is greater than the sum of their individual effects. Modelling multiplicative and additive GxE using linear and logistic regressions has been described elsewhere ${ }^{29,30,40}$.

In the $\mathrm{GxE}$ regression models, we included individuals from UKB with genetic, smoking and psychotic experiences data $(n=143,320$ for any psychotic experience, $n=$ 143,043 for hallucinations, and $n=143,245$ for delusions). Independent variables were the $\mathrm{PRS}_{\mathrm{DEP}}, \mathrm{PRS}_{\mathrm{ADHD}}$, $\mathrm{PRS}_{\mathrm{SCZ}}$ or $\mathrm{PRS}_{\mathrm{BP}}$ for each individual, smoking phenotype, ten principal components and genotyping batch. Dependent variables were lifetime occurrence of hallucinations, delusions or any psychotic experience. Models also included the interaction of smoking and PRS with genotyping batch and the principal components, to control for the effect of these covariates on the interaction term ${ }^{41}$. To examine whether the $\mathrm{GxE}$ was significant after multiple testing correction $(p<0.0021$ based on 24 models under additive and multiplicative models: $0.05 / 24=0.0021$ ), we used the anova function from $\mathrm{R}$ package 'car'. All models were constructed in $\mathrm{R}$ (3.5.1).

\section{Sensitivity analyses}

The inclusion of individuals with psychotic disorders (Supplementary Table 4) may inflate the variance explained by the PRSs and lead to false-positive GxE. Therefore, we repeated the significant interaction effect analysis after excluding individuals that met the criteria for psychotic disorders.

\section{Results}

In all, 157,366 individuals completed the mental health questionnaire. From those, 2067 reported delusions, 6689 hallucinations and 7803 any psychotic experience. In all, 953 individuals had both lifetime delusions and hallucinations. Sample sizes for controls were 155,182, 150,336 and 149,289 for delusions, hallucinations, and any psychotic experience, respectively.

For the smoking variables derived from the full cohort, 273,542 individuals reported that they never smoked, 173,072 were former smokers and 52,979 were current smokers. The mean number of packs per year smoked was 23.4 $(\mathrm{SD}=18.8)$. In all, 126,632 individuals reported that their mothers had smoked around the participant's birth, whereas 306,266 reported the opposite. Table 1 includes demographic, psychiatric and behavioural characteristics of the sample before genetic data QC. Supplementary Table 7 shows the number of individuals with psychotic experiences stratified by smoking status, maternal smoking and mean number of packs smoked per year after genetic data QC.

\section{Smoking and psychotic experiences}

The odds of having delusions, hallucinations or any psychotic experience was significantly higher in current or former tobacco users when compared with non-users, and in participants whose mothers smoked around the time of birth (Table 2). There was a positive linear relationship between the number of packs of cigarettes and risk of psychotic experiences (Table 2). Associations between smoking and the likelihood of having psychotic experiences were strongest for continued tobacco smoking (current smoking vs never smoked). Associations were attenuated after adjustment for diagnosis of psychotic 
Table 1 Demographic, psychiatric and behavioural characteristics of the sample.

\begin{tabular}{|c|c|c|c|c|c|c|}
\hline & \multicolumn{2}{|c|}{ Any psychotic experience } & \multicolumn{2}{|l|}{ Delusions } & \multicolumn{2}{|c|}{ Hallucinations } \\
\hline & $\begin{array}{l}\text { Cases } \\
n=7803\end{array}$ & $\begin{array}{l}\text { Controls } \\
n=149,289\end{array}$ & $\begin{array}{l}\text { Cases } \\
n=2067\end{array}$ & $\begin{array}{l}\text { Controls } \\
n=155,182\end{array}$ & $\begin{array}{l}\text { Cases } \\
n=6689\end{array}$ & $\begin{array}{l}\text { Controls } \\
n=150,336\end{array}$ \\
\hline Age-_[mean years $(\mathrm{SD})]$ & $54.6(7.8)$ & $56(7.7)$ & $53.8(7.9)$ & $55.9(7.7)$ & $54.7(7.8)$ & $56(7.7)$ \\
\hline Males $N$ [\%] & $3082[39.5 \%]$ & $64,940[43.5 \%]$ & $1000[48.4 \%]$ & $67,193[43.3 \%]$ & $2535[37.9 \%]$ & $65,546[43.6 \%]$ \\
\hline \multicolumn{7}{|c|}{ Behavioural/psychiatric during lifetime (Self-reported in Mental Health Questionnaire) } \\
\hline Depression $N[\%]$ & $4627[59.3 \%]$ & $48,071[32.2 \%]$ & $1482[71.7 \%]$ & $51,303[33.06 \%]$ & $3860[57.7 \%]$ & $48,859[32.5 \%]$ \\
\hline ADHD N [\%] & $22[0.28 \%]$ & $100[0.07 \%]$ & $11[0.53 \%]$ & $122[0.08 \%]$ & $15[0.22 \%]$ & $115[0.08 \%]$ \\
\hline Schizophrenia N [\%] & 125 [1.60\%] & $0[0 \%]$ & 109 [5.27\%] & $46[0.03 \%]$ & 100 [1.49\%] & $55[0.04 \%]$ \\
\hline $\begin{array}{l}\text { Mania, hypomania, bipolar or manic-depression } \\
\text { N [\%] }\end{array}$ & 358 [4.59\%] & $423[0.28 \%]$ & 255 [12.34\%] & $573[0.37 \%]$ & 268 [4.00\%] & $556[0.37 \%]$ \\
\hline $\begin{array}{l}\text { Any other type of psychosis or psychotic illness } \\
N[\%]\end{array}$ & 358 [4.59\%] & $0[0 \%]$ & 272 [13.16\%] & 319 [0.21\%] & $270[4.04 \%]$ & $324[0.22 \%]$ \\
\hline \multicolumn{7}{|l|}{ Demographics } \\
\hline Migrant [Yes \%] & $570[7.3 \%]$ & $9554[6.4 \%]$ & 159 [7.7\%] & $9932[6.4 \%]$ & $482[7.2 \%]$ & $9622[6.4 \%]$ \\
\hline Adverse life experiences & $7077[90.7 \%]$ & $114,803[76.9 \%]$ & 1914 [92.6\%] & $120,111[77.4 \%]$ & $6060[90.6 \%]$ & $115,759[77.0 \%]$ \\
\hline \multicolumn{7}{|l|}{ Socio economic status (as per house ownership) } \\
\hline Own outright $N$ [\%] & $3628[46.5 \%]$ & $82,557[55.3 \%]$ & $858[41.5 \%]$ & $85,350[55.0 \%]$ & $3157[47.2 \%]$ & $82,985[55.2 \%]$ \\
\hline Own mortgage $N[\%]$ & 3074 [39.4\%] & $57,028[38.2 \%]$ & 804 [38.9\%] & 59,435 [38.3\%] & 2622 [39.2\%] & $58,330[38.8 \%]$ \\
\hline Rent social N [\%] & $523[6.7 \%]$ & $3583[2.4 \%]$ & 190 [9.2\%] & $4035[2.6 \%]$ & $441[6.6 \%]$ & $3758[2.5 \%]$ \\
\hline Rent private $N$ [\%] & $328[4.2 \%]$ & 3434 [2.3\%] & 132 [6.4\%] & 3569 [2.3\%] & $261[3.9 \%]$ & 3458 [2.3\%] \\
\hline Accommodation rent free $N$ [\%] & 86 [1.1\%] & $896[0.6 \%]$ & 27 [1.3\%] & $931[0.6 \%]$ & 74 [1.1\%] & $902[0.6 \%]$ \\
\hline
\end{tabular}

experiences, depression and ADHD but remained significant. Only packs smoked per year on occurrence of delusions did not pass the significance threshold after multiple testing correction $(p<0.0125)$ (Table 2).

The $\mathrm{PRS}_{\mathrm{SCZ}}, \mathrm{PRS}_{\mathrm{DEP}}, \mathrm{PRS}_{\mathrm{ADHD}}$ and $\mathrm{PRS}_{\mathrm{BP}}$ were significantly associated with psychotic experiences (Fig. 2) and with smoking behaviour (Supplementary Fig. 1). The proportion of variance in psychotic experiences explained by PRSs was small, the highest was for $\mathrm{PRS}_{\mathrm{SCZ}}$, which predicted $0.89 \%$ of the variance for lifetime delusions (Fig. 2a). Results were dependent on the type of psychotic experience: $\mathrm{PRS}_{\mathrm{SCZ}}$ and $\mathrm{PRS}_{\mathrm{BP}}$ explained almost double the percentage of the variance for delusions than for hallucinations (Fig. 2a, d), and p-values for $\mathrm{PRS}_{\mathrm{ADHD}}$ passed multiple testing correction for hallucinations and any psychotic experience, but not for delusions (Fig. 2c).

Therefore, it is possible that genetic risk confounds the relationship between smoking and psychotic experiences. To test this hypothesis, we included $\mathrm{PRS}_{\mathrm{SCZ}}, \mathrm{PRS}_{\mathrm{DEP}}, \mathrm{PRS}_{\mathrm{ADHD}}$ and $\mathrm{PRS}_{\mathrm{BP}}$ as covariates in the analyses. All the regression models (except packs smoked per year on delusions and hallucinations) remained significant after the inclusion of the PRS $_{\mathrm{SCZ}}, \mathrm{PRS}_{\mathrm{DEP}}, \mathrm{PRS}_{\mathrm{ADHD}}$ and PRS $\mathrm{BP}$ (Table 2).
Interaction between genetic risk of psychiatric disorders and smoking on psychotic experiences

We found significant additive interactions between $\mathrm{PRS}_{\mathrm{ADHD}}$ and PRS $\mathrm{DEP}_{\mathrm{DEP}}$ (but not PRS $\mathrm{PCZ}_{\mathrm{SCZ}}$ and $\mathrm{PRS}_{\mathrm{BP}}$ ) and smoking status (current vs never smoker) on reporting lifetime delusions. That is, the combined effect of PRS $_{\mathrm{ADHD}}$ and smoking, or PRS $\mathrm{DEP}_{\text {and smoking was sig- }}$ and nificantly greater than the sum of their individual effects (Fig. 3 and Supplementary Table 8 for OR and $\beta$ values). However, there were no significant interactions between PRSs and smoking status for hallucinations or any psychotic experiences (Fig. 3).

To ensure that the significant interaction for PRS $_{\mathrm{ADHD}}$ and PRS ticipants with a diagnosis of bipolar disorder or schizophrenia, we excluded individuals meeting criteria for diagnosis of psychotic disorders (Supplementary Table 9) and repeated the GxE analyses. All the interaction terms remained significant (Supplementary Fig. 2 and Supplementary Table 10).

There were no significant interactions between the PRSs and maternal smoking, nor between the PRSs and number of packs per year (Supplementary Tables 11 and 12). 
Table 2 Phenotypic relationships between smoking and psychotic experiences within UKB. Logistic regression models were used to assess the association between smoking and three psychotic experiences phenotypes.

\begin{tabular}{|c|c|c|c|c|c|c|}
\hline & \multicolumn{2}{|l|}{ Unadjusted $^{a}$} & \multicolumn{2}{|c|}{$\begin{array}{l}\text { Adjusted for psychiatric } \\
\text { disorders }^{b}\end{array}$} & \multicolumn{2}{|c|}{$\begin{array}{l}\text { Adjusted for psychiatric } \\
\text { disorders andPRSs }\end{array}$} \\
\hline & OR $(95 \% \mathrm{Cl})$ & $p$ & OR $(95 \% \mathrm{Cl})$ & $p$ & OR $(95 \% \mathrm{Cl})$ & $p$ \\
\hline \multicolumn{7}{|l|}{ Any psychotic experience } \\
\hline Former vs never smoking & $1.22(1.16-1.28)$ & $4.99 \times 10^{-14}$ & $1.16(1.10-1.22)$ & $2.89 \times 10^{-8}$ & $1.15(1.09-1.21)$ & $2.87 \times 10^{-7}$ \\
\hline Current vs never smoking & $1.76(1.62-1.91)$ & $4.76 \times 10^{-42}$ & $1.47(1.35-1.60)$ & $3.76 \times 10^{-19}$ & $1.45(1.33-1.58)$ & $1.08 \times 10^{-17}$ \\
\hline Maternal smoking & $1.26(1.19-1.33)$ & $5.89 \times 10^{-17}$ & $1.20(1.14-1.27)$ & $5.87 \times 10^{-11}$ & $1.19(1.13-1.26)$ & $6.98 \times 10^{-10}$ \\
\hline Packs smoked per year & 1.006 (1.004-1.008) & $4.61 \times 10^{-7}$ & $1.004(1.001-1.006)$ & $2.95 \times 10^{-3}$ & $1.003(1.001-1.006)$ & $7.17 \times 10^{-3}$ \\
\hline \multicolumn{7}{|l|}{ Delusions } \\
\hline Former vs never smoking & $1.28(1.16-1.41)$ & $1.68 \times 10^{-6}$ & $1.18(1.07-1.31)$ & $1.29 \times 10^{-3}$ & $1.17(1.05-1.29)$ & $3.90 \times 10^{-3}$ \\
\hline Current vs never smoking & $2.21(1.9-2.55)$ & $9.74 \times 10^{-27}$ & $1.61(1.38-1.87)$ & $9.41 \times 10^{-10}$ & $1.57(1.35-1.83)$ & $8.13 \times 10^{-9}$ \\
\hline Maternal smoking & $1.28(1.15-1.42)$ & $3.17 \times 10^{-6}$ & $1.20(1.08-1.34)$ & $8.09 \times 10^{-4}$ & $1.19(1.06-1.32)$ & $2.06 \times 10^{-3}$ \\
\hline Packs smoked per year & 1.009 (1.005-1.013) & $4.73 \times 10^{-6}$ & 1.005 (1.000-1.009) & 0.0383 & $1.004(0.999-1.008)$ & 0.0740 \\
\hline \multicolumn{7}{|l|}{ Hallucinations } \\
\hline Former vs never smoking & $1.21(1.14-1.28)$ & $2.66 \times 10^{-11}$ & $1.15(1.09-1.22)$ & $8.05 \times 10^{-7}$ & $1.14(1.08-1.21)$ & $4.76 \times 10^{-6}$ \\
\hline Current vs never smoking & $1.76(1.61-1.92)$ & $8.08 \times 10^{-37}$ & $1.49(1.36-1.63)$ & $2.36 \times 10^{-18}$ & $1.47(1.34-1.61)$ & $4.86 \times 10^{-17}$ \\
\hline Maternal smoking & $1.26(1.19-1.34)$ & $4.47 \times 10^{-15}$ & $1.21(1.14-1.28)$ & $4.66 \times 10^{-10}$ & $1.20(1.13-1.27)$ & $3.92 \times 10^{-9}$ \\
\hline Packs smoked per year & 1.005 (1.003-1.008) & $1.77 \times 10^{-5}$ & $1.003(1.001-1.006)$ & 0.0101 & $1.003(1.001-1.006)$ & 0.0185 \\
\hline 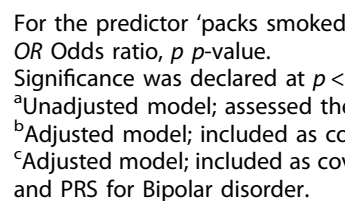 & $\begin{array}{l}\text { year', OR represents } \\
25 \text { after Bonferroni c }\end{array}$ & $\begin{array}{l}\text { tic disorders, } \\
\text { ic disorders, d }\end{array}$ & $\begin{array}{l}\text { xperiences and smokir } \\
\text { ID and depression. }\end{array}$ & $\begin{array}{l}\text { phenotype). } \\
\text { sing logistic re }\end{array}$ & ssion models. & 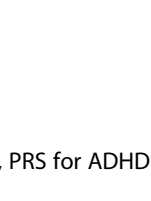 \\
\hline
\end{tabular}

\section{Discussion}

Our study investigated whether smoking is associated with the occurrence of psychotic experiences independently from diagnosis of psychotic disorders, depression or ADHD, or genetic predisposition for these disorders. We also tested whether the effects of smoking were modulated by the genetic risk in gene-environment interaction models. We found smoking status, maternal smoking and number of packs smoked per year were all significantly associated with increased risk of psychotic experiences. GxE modelling indicated the effects of two PRSS (DEP \& ADHD) on delusions was significantly greater in current smokers compared to never smokers. These results suggest both genetic risk and smoking behaviour contribute to specific types of psychotic experiences.

A few previous studies have assessed PRS-environment interactions on psychotic experiences finding that higher genetic risk exacerbates environmental effects. However, these studies have focused exclusively on genetic risk of schizophrenia and environmental factors such as stress ${ }^{42}$ and birth weight ${ }^{43}$. Our study is the first one to explore
PRS by environment interactions with smoking on psychotic experiences and the first to explore these effects in the context of genetic risk of depression, bipolar disorder and ADHD. Further, our study had greater power than previous ones because we used at least 30 times larger sample sizes, we calculated multiple PRSs at higher $p$ values that increased the predictive power of the genetic score, and we used the largest GWAS summary statistics available to date.

The interaction of smoking status with $\mathrm{PRS}_{\mathrm{DEP}}$, but not with $\mathrm{PRS}_{\mathrm{SCZ}}$ or $\mathrm{PRS}_{\mathrm{BP}}$, on predicting delusions is of particular interest. Delusions of reference and persecution are classified as some of the core symptoms of psychotic disorders (i.e. schizophrenia and bipolar disorder) but they are rarely part of the diagnostic criteria for depres$\operatorname{sion}^{44}$. Wooton et al. ${ }^{14}$ reported that genetic risk for depression increased smoking, but there was unclear evidence for genetic risk for schizophrenia increasing smoking. Our finding that $\mathrm{PRS}_{\mathrm{DEP}}$ (but not $\mathrm{PRS}_{\mathrm{SCZ}}$ or $\mathrm{PRS}_{\mathrm{BP}}$ ) interacts with smoking on predicting psychotic experiences points at a stronger relationship between 


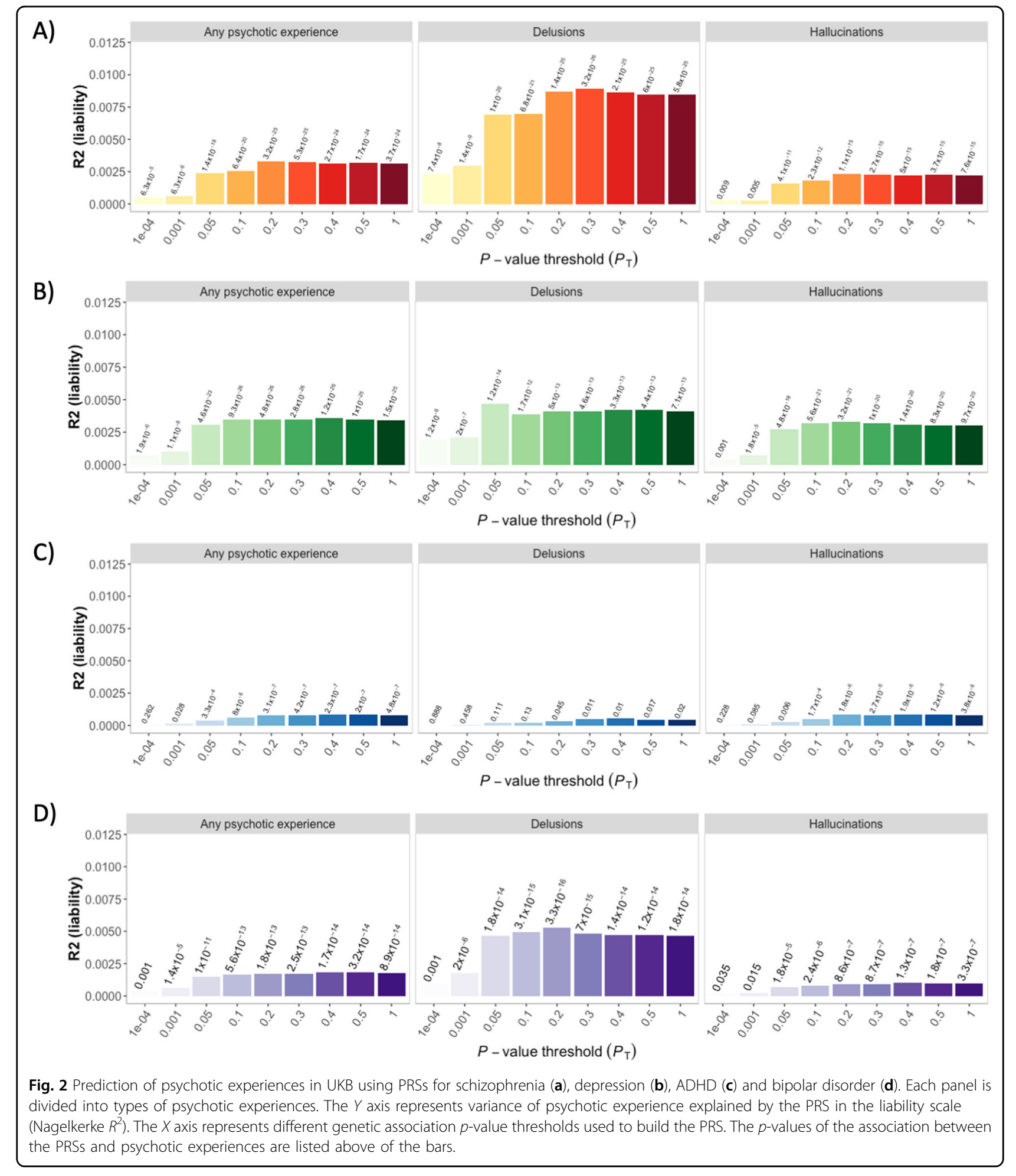

smoking and depression than between smoking and schizophrenia or between smoking and bipolar disorder. Our sensitivity analyses, where the interaction remained significant after excluding people with psychotic disorders, supports this hypothesis.
In line with previous studies, we found significant associations between the smoking phenotypes and psychotic experiences $^{10-13,17}$, and between $\mathrm{PRS}_{\mathrm{SCZ}}, \mathrm{PRS}_{\mathrm{DEP}}$, $\mathrm{PRS}_{\mathrm{ADHD}}$ and $\mathrm{PRS}_{\mathrm{BP}}$ and the smoking phenotypes and psychotic experiences ${ }^{25,36,45,46}$. These findings suggest 
Any psychotic experience

A)

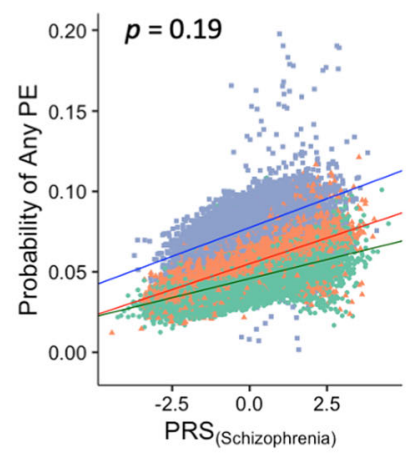

B)

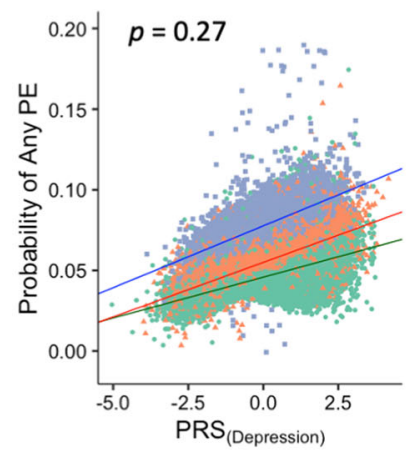

C)

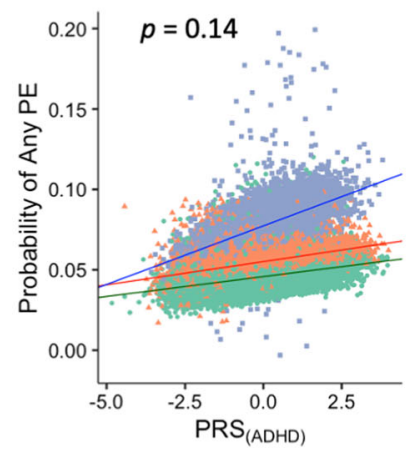

D)

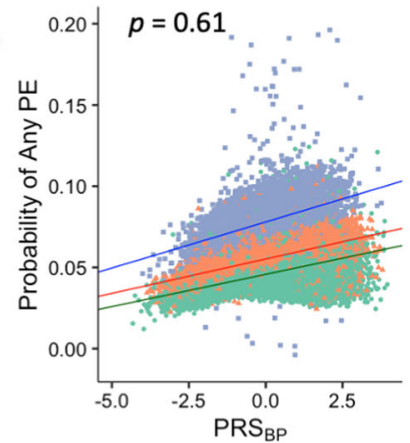

Delusions
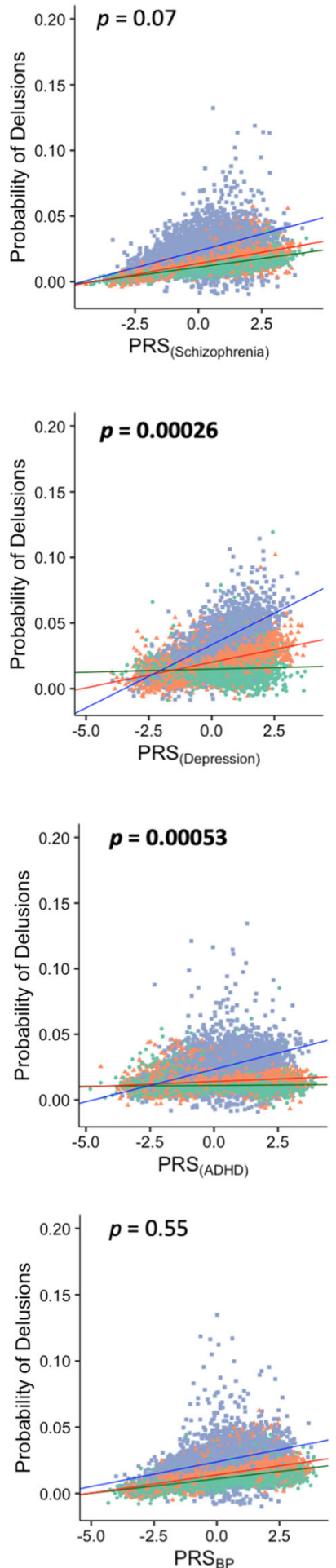

Hallucinations
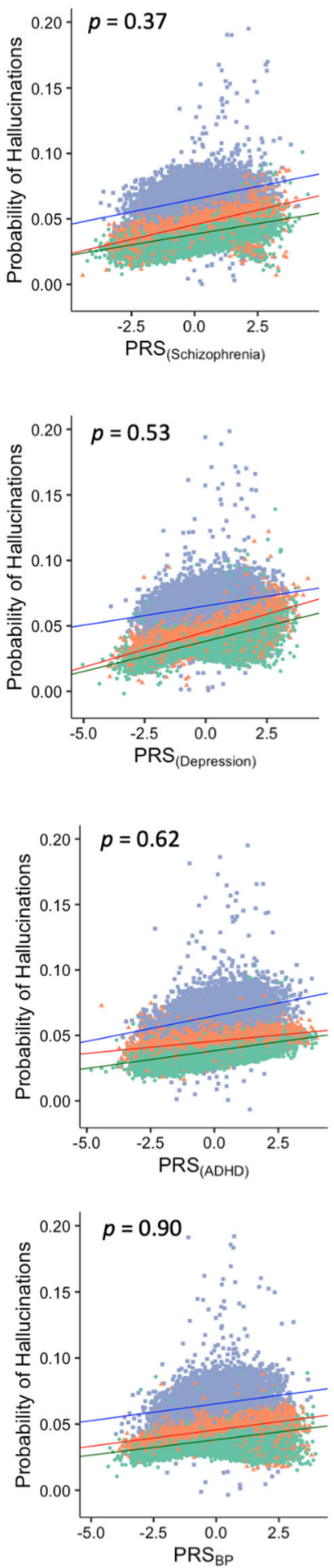

\section{Smoking Status - Never ^ Former " Current}

Fig. 3 Associations between polygenic risk score for a schizophrenia $\left(\mathrm{PRS}_{\mathrm{SCZ}}\right)$, b depression $\left(\mathrm{PRS}_{\mathrm{DEP}}\right), \mathbf{c} \mathrm{ADHD}(\mathrm{PRS}$ ADHD $)$ and $\mathbf{d}$ bipolar disorder $\left(\mathrm{PRS} \mathrm{BP}_{\mathrm{BP}}\right)$ and occurrence of psychotic experiences (PE) under the additive model. Current smokers are shown as blue squares, former smokers as orange triangles, and never smokers as green circles. $P$ values for the PRS $\times$ smoking status interactions are shown on the upper part of each plot. Significant associations ( $p<0.0021$ based on 24 tests; 12 additive interactions and 12 multiplicative interactions) are highlighted in bold. The effects of PRS DEP and PRS ${ }_{A D H D}$ on the probability of having delusions are greater among current smokers than former and never smokers. 
that the relationship between the smoking variables and psychotic experiences may be confounded by shared genetic influences. However, the effects of smoking remained very similar after adjusting for PRSs, providing little evidence of genetic confounding by common genetic variation associated with schizophrenia, depression, ADHD and bipolar disorder. Previous studies have shown that the effects of smoking on psychotic experiences remain significant after adjustment for psychiatric disorders $^{16}$. However, this is the first study to show these effects also remain significant after adjustment for genetic risk of specific psychiatric disorders.

The effects of maternal smoking after adjusting for the PRSs are also interesting. Previous within-family studies suggest that, once unmeasured familial factors are taken into account, maternal smoking has little effect on psychotic disorders ${ }^{20}$. In the current study, the effects of maternal smoking persisted following adjustment for $\mathrm{PRS}_{\mathrm{SCZ}}, \mathrm{PRS}_{\mathrm{DEP}} \mathrm{PRS}_{\mathrm{ADHD}}$ and $\mathrm{PRS}_{\mathrm{BP}}$. This tentatively suggests that genetic risk for these disorders does not confound the relationship between maternal smoking and psychotic experiences. Nevertheless, the PRSs tested only capture a small fraction of the genetic risk for psychiatric disorders. Replication of these effects using more powerful estimates of genetic risk will be required to test this hypothesis.

There are some limitations of this study: it is retrospective meaning it is subject to recalling biases, which occur when participants do not accurately remember past experiences, and this can lead to spurious results. We have relied on self-reported data, which might be affected by different conceptions of what it is a psychotic experience. There were 351 individuals that completed the UKB mental health questionnaire that may have also participated in the Psychiatric Genomic Consortium cohorts analysed by Wray et al. ${ }^{37}$. The sample overlap was minimal, with $0.079 \%$ for the discovery sample $(351 / 446,238)$, and $0.242 \%$ for the target sample $(351 / 144,818)$. The overlap was identified using a checksum-based approach as described in Howard et al. ${ }^{47}$ and the impact over this overlap is unknown. We reported an interaction between genetic risk and smoking, but the percentage of variance explained by the PRSs was very low $(<1 \%)$, therefore further work is required to understand the remaining sources of phenotypic variance. There is also evidence of a 'healthy volunteer' bias in $\mathrm{UKB}^{48}$, especially among people who completed the mental health questionnaire ${ }^{31}$, which may make this study not fully representative of the general population. Finally, the present study was focused on individuals of European ancestry therefore these results might not be valid for other ancestries ${ }^{49}$.

Despite the limitations, we report for the first time that smoking is associated with occurrence of psychotic experiences after adjusting for genetic risk for mental health and that current smokers with higher genetic risk for depression and ADHD are more likely to experience delusions but not hallucinations. These results emphasise the importance of assessing environmental and genetic factors jointly and separately, and exploring particular types of psychotic experiences, as they can be differentially associated with smoking or other risk factors. This study also highlights the relationship between smoking and depression, and between smoking and ADHD on the occurrence of specific types of psychotic experiences in the general population, encouraging further studies to explore the effect of genetic risk for other psychiatric disorders on psychosis. Taken together, our results support the distinction of types of psychotic symptoms, the inclusion of environmental factors, and the study of crossdisorder genetic predictors to inform biological mechanisms underlying psychotic experiences.

\begin{abstract}
Acknowledgements
This paper is dedicated to the memory of our dear friend and colleague Rob Keers, who sadly passed away while this paper was being peer-reviewed. We thank the UK Biobank participants who donated their time, experiences and DNA to this research, and to the UK Biobank team for making the data available. J.G-G. would like to thank Dr. Jonathan Coleman, Dr. Christopher Hübel and Olakunle Oginni for valuable feedback on the analyses and the paper draft. J.G-G. acknowledges support from the Queen Mary Principal's Research Studentship in the School of Biological and Chemical Sciences. D.M.H. is supported by a Sir Henry Wellcome Postdoctoral Fellowship (Reference 213674/Z/18/Z) and a 2018 NARSAD Young Investigator Grant from the Brain \& Behavior Research Foundation (Ref: 27404). P.B.M. acknowledges support from the National Institutes of Health Research (NIHR) Cardiovascular Biomedical Centre at Barts and The London, Queen Mary University of London (QMUL). J.R. acknowledges support from the European Union's Horizon 2020 research and innovation programme under the Marie Sklodowska-Curie grant agreement No 786833. C.H.B. acknowledges support from NIH grant No UO1 DA04440001A1. C.H.B. is a member of the Royal Society Industry Fellows' College. R.K. is supported by Wellcome Trust Grant 208881/Z/17/Z.
\end{abstract}

\section{Author details}

${ }^{1}$ School of Biological and Chemical Sciences, Queen Mary University of London, London E1 4NS, UK. ${ }^{2}$ Clinical Pharmacology, William Harvey Research Institute, Barts and The London School of Medicine and Dentistry, Queen Mary University of London, London EC1M 6BQ, UK. ${ }^{3}$ Social, Genetic and Developmental Psychiatry Centre, Institute of Psychiatry, Psychology and Neuroscience, King's College London, London, UK. ${ }^{4}$ Division of Psychiatry, University of Edinburgh, Royal Edinburgh Hospital, Edinburgh, UK. ${ }^{5} \mathrm{NIHR}$ Barts Cardiovascular Biomedical Research Centre, Barts and The London School of Medicine and Dentistry, Queen Mary University of London, London EC1M 6BQ, UK

\section{Code availability}

Analytical code used to define outcomes and regression models for this project is available at https://github.com/juditperala/PRSxSmoking.

\section{Conflict of interest}

The authors declare that they have no conflict of interest.

\section{Publisher's note}

Springer Nature remains neutral with regard to jurisdictional claims in published maps and institutional affiliations.

Supplementary Information accompanies this paper at (https://doi.org/ 10.1038/s41398-020-01009-8). 
Received: 19 January 2020 Revised: 21 August 2020 Accepted: 3 September 2020

Published online: 28 September 2020

\section{References}

1. McGrath, J. J. et al. Psychotic experiences in the general population: a crossnational analysis based on 31,261 respondents from 18 countries. JAMA Psychiatry 72, 697-705 (2015).

2. Zavos, H. M. S. et al. Consistent etiology of severe, frequent psychotic experiences and milder, less frequent manifestations: a twin study of specific psychotic experiences in adolescence. JAMA Psychiatry 71, 1049-1057 (2014).

3. Ronald, A. Recent quantitative genetic research on psychotic experiences: new approaches to old questions. Curr. Opin. Behav. Sci. 2, 81-88 (2015).

4. Sieradzka, D. et al. Heritability of individual psychotic experiences captured by common genetic variants in a community sample of adolescents. Behav. Genet. 45, 493-502 (2015).

5. Ronald, A. \& Pain, O. A systematic review of genome-wide research on psychotic experiences and negative symptom traits: new revelations and implications for psychiatry. Hum. Mol. Genet 27, R136-R152 (2018).

6. Zavos, H. M. S. et al. Shared etiology of psychotic experiences and depressive symptoms in adolescence: a longitudinal twin study. Schizophr. Bull. 42, 1197-1206 (2016).

7. Legge, S. E. et al. Association of genetic liability to psychotic experiences with neuropsychotic disorders and traits. JAMA Psychiatry. https://doi.org/10.1001/ jamapsychiatry.2019.2508 (2019)

8. Myles, N. et al. Tobacco use before, at, and after first-episode psychosis: a systematic meta-analysis. J. Clin. Psychiatry 73, 468-475 (2012).

9. Gurillo, P., Jauhar, S., Murray, R. M. \& MacCabe, J. H. Does tobacco use cause psychosis? Systematic review and meta-analysis. Lancet Psychiatry 2, 718-725 (2015).

10. Kendler, K. S., Lönn, S. L., Sundquist, J. \& Sundquist, K. Smoking and schizophrenia in population cohorts of Swedish women and men: a prospective corelative control study. Am. J. Psychiatry 172, 1092-1100 (2015).

11. Weiser, M. et al. Higher rates of cigarette smoking in male adolescents before the onset of schizophrenia: a historical-prospective cohort study. Am. J. Psychiatry 161, 1219-1223 (2004).

12. Krishnadas, R., Jauhar, S., Telfer, S., Shivashankar, S. \& McCreadie, R. G. Nicotine dependence and illness severity in schizophrenia. Br. J. Psychiatry 201, 306-312 (2012).

13. Gage, S. H. et al. Associations of cannabis and cigarette use with psychotic experiences at age 18: findings from the avon longitudinal study of parents and children. Psychol. Med. 44, 3435-3444 (2014).

14. Wootton, R. E. et al. Evidence for causal effects of lifetime smoking on risk for depression and schizophrenia: a Mendelian randomisation study. Psychol. Med. https://doi.org/10.1017/S0033291719002678 (2019)

15. Mustonen, A. et al. Smokin' hot: adolescent smoking and the risk of psychosis. Acta Psychiatr. Scand. 138, 5-14 (2018).

16. Degenhardt, L. et al. The associations between psychotic experiences, and substance use and substance use disorders: findings from the World Health Organisation World Mental Health Surveys. Addiction 113, 924-934 (2018).

17. Bhavsar, V. et al. Tobacco smoking is associated with psychotic experiences in the general population of South London. Psychol. Med. 48, 123-131 (2018).

18. Oh, H. Y., Koyanagi, A., Singh, F. \& DeVylder, J. Is smoking tobacco associated with psychotic experiences across racial categories in the United States? Findings from the Collaborative Psychiatric Epidemiological Surveys. Psychiatry Res. 246, 58-61 (2016).

19. Mackay, D. F., Anderson, J. J., Pell, J. P., Zammit, S. \& Smith, D. J. Exposure to tobacco smoke in utero or during early childhood and risk of hypomania: Prospective birth cohort study. Eur. Psychiatry 39, 33-39 (2017).

20. Quinn, P. D. et al. Association between maternal smoking during pregnancy and severe mental illness in offspring. JAMA Psychiatry 74, 589-596 (2017).

21. Jones, H. J. et al. Association of combined patterns of tobacco and cannabis use in adolescence with psychotic experiences. JAMA Psychiatry 75, 240-246 (2018).

22. van Gastel, W. A. et al. Cigarette smoking and cannabis use are equally strongly associated with psychotic-like experiences: a cross-sectional study in 1929 young adults. Psychol. Med. 43, 2393-2401 (2013).
23. de Leon, J. \& Diaz, F. J. A meta-analysis of worldwide studies demonstrates an association between schizophrenia and tobacco smoking behaviors. Schizophr. Res. 76, 135-157 (2005).

24. Hartz, S. M. et al. Association between substance use disorder and polygenic liability to schizophrenia. Biol. Psychiatry 82, 709-715 (2017).

25. Reginsson, G. W. et al. Polygenic risk scores for schizophrenia and bipolar disorder associate with addiction. Addiction Biol. 23, 485-492 (2018).

26. Carey, C. E. et al. Associations between polygenic risk for psychiatric disorders and substance involvement. Front Genet 7, 149 (2016).

27. Du Rietz, Eetal Association of polygenic risk for attention-deficit/hyperactivity disorder with co-occurring traits and disorders. Biol. Psychiatry Cogn. Neurosci. Neuroimaging 3, 635-643 (2018).

28. Peyrot, W. J. et al. Effect of polygenic risk scores on depression in childhood trauma. Br. J. Psychiatry 205, 113-119 (2014).

29. Mullins, N. et al. Polygenic interactions with environmental adversity in the aetiology of major depressive disorder. Psychol. Med. 46, 759-770 (2016).

30. Coleman, J. R. I. et al. Genome-wide gene-environment analyses of major depressive disorder and reported lifetime traumatic experiences in UK Biobank. bioRxiv 247353. https://doi.org/10.1101/247353 (2018)

31. Davis, K. A. S. et al. Mental health in UK Biobank-development, implementation and results from an online questionnaire completed by 157366 participants: a reanalysis. BJPsych Open 6, e18 (2020).

32. Bycroft, $C$. et al. The UK Biobank resource with deep phenotyping and genomic data. Nature 562, 203-209 (2018).

33. Ramírez, J. et al. Thirty loci identified for heart rate response to exercise and recovery implicate autonomic nervous system. Nat. Commun. 9, 1947 (2018).

34. Hanscombe, K. B., Coleman, J. R. I., Traylor, M. \& Lewis, C. M. ukbtools: An R package to manage and query UK Biobank data. PLOS ONE 14, e0214311 (2019).

35. Choi, S. W. \& O'Reilly, P. F. PRSice-2: polygenic risk score software for biobankscale data. Gigascience. 8, gizO82 (2019).

36. Demontis, D. et al. Discovery of the first genome-wide significant risk loci for attention deficit/hyperactivity disorder. Nat. Genet 51, 63-75 (2019).

37. Wray, N. R. et al. Genome-wide association analyses identify 44 risk variants and refine the genetic architecture of major depression. Nat. Genet $\mathbf{5 0}$, 668-681 (2018).

38. Ripke, S. et al. Biological insights from 108 schizophrenia-associated genetic loci. Nature 511, 421-427 (2014).

39. Stahl, E. A. et al. Genome-wide association study identifies 30 loci associated with bipolar disorder. Nat. Genet. 51, 793-803 (2019).

40. Trotta, A. et al. Interplay between schizophrenia polygenic risk score and childhood adversity in first-presentation psychotic disorder: a pilot study. PLOS ONE 11, e0163319 (2016).

41. Keller, M. C. Gene $\times$ environment interaction studies have not properly controlled for potential confounders: the problem and the (simple) solution. Biol. Psychiatry 75, 18-24 (2014).

42. Hatzimanolis, A. et al. Stress-dependent association between polygenic risk for schizophrenia and schizotypal traits in young army recruits. Schizophr. Bull. 44, 338-347 (2018).

43. Liuhanen, J. et al. Interaction between compound genetic risk for schizophrenia and high birth weight contributes to social anhedonia and schizophrenia in women. Psychiatry Res. 259, 148-153 (2018).

44. Diagnostic and Statistical Manual of Mental Disorders (DSM-5 ${ }^{\circledR}$ ). https://www. appi.org/Diagnostic_and_Statistical_Manual_of_Mental_Disorders_DSM5_Fifth_Edition (2013)

45. Howard, D. M. et al. Genome-wide association study of depression phenotypes in UK Biobank identifies variants in excitatory synaptic pathways. Nat. Commun. 9, 1-10 (2018).

46. Liu, M. et al. Association studies of up to 1.2 million individuals yield new insights into the genetic etiology of tobacco and alcohol use. Nat. Genet. https://doi.org/10.1038/s41588-018-0307-5 (2019)

47. Howard, D. M. et al. Genome-wide meta-analysis of depression identifies 102 independent variants and highlights the importance of the prefrontal brain regions. Nat. Neurosci. 22, 343-352 (2019).

48. Fry, A. et al. Comparison of sociodemographic and health-related characteristics of UK Biobank participants with those of the general population. Am. J. Epidemiol. 186, 1026-1034 (2017).

49. Duncan, L. E. et al. Analysis of polygenic risk score usage and performance in diverse human populations. Nat. Commun. 10, 3328 (2019). 